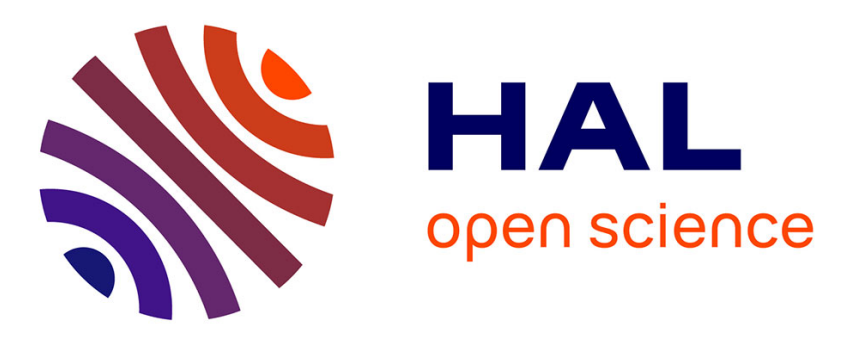

\title{
Energy efficient downlink massive MIMO: Is 1-bit quantization a solution?
}

Alexandre Marcastel, Inbar Fijalkow, Lee Swindlehurst

\section{To cite this version:}

Alexandre Marcastel, Inbar Fijalkow, Lee Swindlehurst. Energy efficient downlink massive MIMO: Is 1-bit quantization a solution?. International Symposium on Wireless Communication Systems, Aug 2019, Oulu, Finland. hal-02189110

\section{HAL Id: hal-02189110 https://hal.science/hal-02189110}

Submitted on 19 Jul 2019

HAL is a multi-disciplinary open access archive for the deposit and dissemination of scientific research documents, whether they are published or not. The documents may come from teaching and research institutions in France or abroad, or from public or private research centers.
L'archive ouverte pluridisciplinaire HAL, est destinée au dépôt et à la diffusion de documents scientifiques de niveau recherche, publiés ou non, émanant des établissements d'enseignement et de recherche français ou étrangers, des laboratoires publics ou privés. 


\title{
Energy efficient downlink massive MIMO: Is 1-bit quantization a solution ?
}

\author{
Alexandre Marcastel $^{1}$, Inbar Fijalkow ${ }^{1}$, Lee Swindlehurst ${ }^{2}$ \\ ${ }^{1}$ ETIS, Université Paris Seine, Université Cergy-Pontoise, ENSEA, CNRS, F-95000 Cergy, France \\ ${ }^{2}$ EECS, University of California at Irvine, CA, USA
}

\begin{abstract}
Massive MIMO aims to build wireless base stations with hundreds of coherently operating antennas serving tens of single antenna users in order to improve both the transmission capacity by a factor 10-50 and the energy-efficiency trade-off by up to a thousand times. Precoding at the base station has been proposed to efficiently implement digital beamforming. It implies a high signal dynamic range and therefore a power backoff resulting in less energy-efficiency. One-bit quantized Zero-Forcing precoding has been proposed to efficiently handle the RF front-end when the array is implemented with so many antennas. In this paper, we analyze the energy-efficiency of the quantized Zero-Forcing precoded systems for a large number of users and a massive MIMO base station.
\end{abstract}

\section{INTRODUCTION}

Massive MIMO base stations with hundreds of coherently operating antennas serving tens of single antenna users is one of the key technologies to enable next-generation wireless communications to improve both the transmission capacity by a factor 10-50 and the energy-efficiency trade-off by up to a thousand times [1].

Precoding at the base station (BS) has been proposed to efficiently implement digital beamforming [2]. However, Zero-Forcing (ZF) or Minimum Mean Square Error (MMSE) precoding leads to a high signal dynamic range and therefore a power backoff resulting in less efficient power amplifiers and a reduction in the energy-efficiency of the system. Since the power consumption and hardware costs increase also with the number of antennas [3], the energy-efficiency of massive MIMO base stations can be questioned. One-bit quantization, namely constant envelope signals using one-bit on both the I and $\mathrm{Q}$ dimensions, has been proposed to suppress the signal dynamic range in order to maximize the power amplifier efficiency, to eliminate the adaptive gain controller and at the same time to minimize the ADC/DAC power consumption [4].

Comparisons between the quantized and unquantized systems have been performed in terms of channel capacity [5][6], bit error rate performance [7] and channel estimation [8]. In addition, energy and spectral efficiency are studied in [9] and [10] but only for the uplink of MIMO system with mixedADC implementations. However, to the best of our knowledge there is no such comparison in terms of energy-efficiency for the downlink. The energy-efficiency of multi-user massive MIMO with full signal dynamic range has been studied in

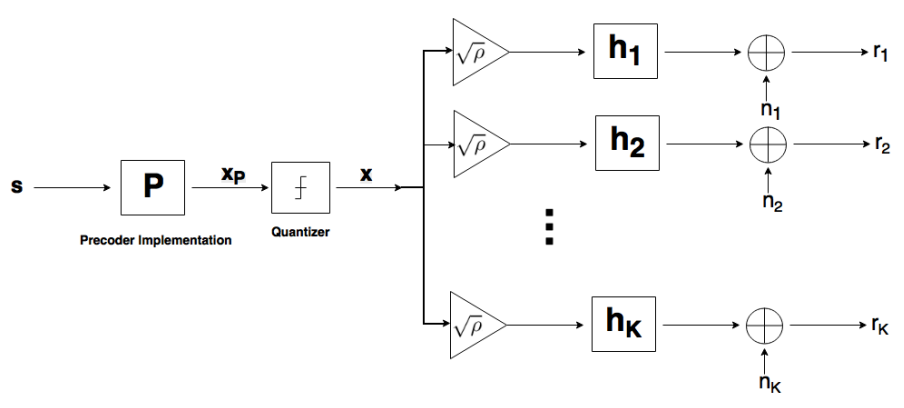

Fig. 1: One-bit quantized system model

[11] and [12] showing the advantage of ZF and MMSE over maximum ratio combining and the quasi logarithmic behavior with respect to the number of antennas. The work in [11] considers only the radiated power, whereas [12] proposes a more realistic power consumption model.

In this paper, we analyze the energy-efficiency of the massive MIMO downlink for both one-bit quantized and full dynamic-range precoded signals. For the sake of simplicity, we focus on the quantized Zero-Forcing precoder and we use random matrix theory to obtain simple approximations with respect to a large number of antennas at the $\mathrm{BS}$ and a large number of users.

\section{Notation}

In what follows, uppercase boldface letters, $\mathbf{A}$, indicate a matrix. Lower boldface letters, a, indicate a column vector, with $a_{k}$ denoting the $k^{\text {th }}$ element of the column vector. The symbols $(.)^{*},(.)^{T}$ and $(.)^{H}$ denote the complex conjugate, matrix transpose and the transpose-conjugate of the argument respectively.

\section{Downlink Massive-Mimo System Model}

\section{A. Downlink Massive-MIMO Models}

We assume a flat-fading downlink scenario in which an $M$-antenna BS is attempting to send QPSK symbols $s_{k}$ to $k=1, \cdots, K$ single-antenna users. The one-bit quantized BS transmits an $M \times 1$ vector $\sqrt{\rho} \mathbf{x}$, where $\sqrt{\rho}$ is a fixed gain (see Fig. 1):

$$
\mathbf{x}=\mathcal{Q}\left(\mathbf{x}_{Z F}\right)=\mathcal{Q}(\mathbf{G s})=\mathcal{Q}\left(\mathcal{R}\left(\mathbf{x}_{Z F}\right)\right)+j \mathcal{Q}\left(\mathcal{I}\left(\mathbf{x}_{Z F}\right)\right)
$$

where the full dynamic range signal is denoted $\mathbf{x}_{Z F}=\mathbf{G s}$ with $\mathbf{G}=\mathbf{H}^{H}\left(\mathbf{H H}^{H}\right)^{-1}$, the ZF precoder corresponding 
to channel $\mathbf{H}$. The quantization operator $\mathcal{Q}$ constrains the elements of $\mathrm{x}$ to be equal to $\pm 1 \pm j$, i.e. one-bit quantization of the in-phase and quadrature components of the signal at the $\mathrm{BS}$, where $\mathcal{R}$ (resp. $\mathcal{I}$ ) indicates the real (resp. imaginary part). We denote $\mathbf{s}=\left[\begin{array}{lll}s_{1} & \cdots & s_{K}\end{array}\right]^{T}$ as the symbols to be transmited to the $K$ users and $\mathcal{S}=\{1+j, 1-j,-1+j,-1-j\}$ the set of QPSK constellation points.

The BS without one-bit quantization corresponds to directly transmitting $\sqrt{\rho_{Z F}} \mathbf{x}_{Z F}$ instead of $\sqrt{\rho} \mathbf{x}$. Unlike [12], no user specific power allocation is performed here.

Let $r_{k}=\mathbf{h}_{k} \mathbf{x}+n_{k}$ be the signal received by user $k$ where $\mathbf{h}_{k}$ represents the Rayleigh channel from the massive MIMO $\mathrm{BS}$ to user $k$, see Fig. 1. For simplicity, we write the received signals from all users in a single vector $\mathbf{r}=\left[\begin{array}{lll}r_{1} & \cdots & r_{K}\end{array}\right]^{T}$ so that the overall system model becomes $\mathbf{r}=\sqrt{\rho} \mathbf{H x}+\mathbf{n}$ where the $K \times 1$ vector $\mathbf{n}$ represents a vector of independent complex isotropic Gaussian noise terms of variance $\sigma_{n}^{2}$ at each user. $\mathbf{H}$ is the $K \times M$ channel matrix with $k^{\text {th }}$ row $\mathbf{h}_{k}$. We will assume that the channel $\mathbf{H}$ is known at the BS.

In the non-quantized case, the received signal is

$$
\mathbf{r}_{Z F}=\sqrt{\rho_{Z F}} \mathbf{H x}_{Z F}+\mathbf{n}=\sqrt{\rho_{Z F}} \mathbf{s}+\mathbf{n}
$$

\section{B. Energy-Efficiency}

The common definition of energy-efficiency (EE) in [bit/Joule] is:

$$
\mathrm{EE}=\frac{\text { Bit rate [bit/s] }}{\text { Energy consumption [Joule/s] }}
$$

Assuming each user considers all multi-user interference and quantization error as independent Gaussian noise, the multiuser MIMO data rate on band $B$ is [13]:

$$
r_{B}=B \sum_{k=1}^{K} \log _{2}\left(1+\mathrm{SQINR}_{k}\right)
$$

where the signal-to-quantization, interference and noise ratio at user $k, \mathrm{SQINR}_{k}$ has been studied in [7]. Note that, even though the signals are not Gaussian we still use the Shannon capacity as an approximation of the rate. [7] allows us to have some insight on the behavior of the quantized approach. We also remark that this data rate is smaller than the sum channel capacity that could be achieved with a different power allocation and more complex receivers.

Given large enough values of $M$ and $K$, and finite $\gamma=$ $\frac{M}{K}>1$, random matrix theory can be used to show that the one-bit quantized SQINR $k$ is approximately (see [7] for details and more general expressions)

$$
\operatorname{SQINR}(\gamma) \approx \frac{\frac{2(\gamma-1)^{2}}{\gamma \pi}}{\left(1-\frac{2}{\pi}\right)\left(1-\frac{1}{\gamma}\right)+\frac{\sigma_{n}^{2}}{2 \rho \sigma^{2}}},
$$

where $\sigma^{2}$ is the variance of the channel gains, which is assumed, for simplicity, to be equal for all users. The SQINR for the unquantized $\mathrm{ZF}$ precoder is simply $\mathrm{SNR}=\rho_{Z F} \frac{2}{\sigma_{n}^{2}}$ since in principle there is no quantization noise and the multiuser interference is eliminated.

In the sequel, we will replace the expression for $\mathrm{SQINR}_{k}$ (5) in (4) and express the energy comsumption with respect to

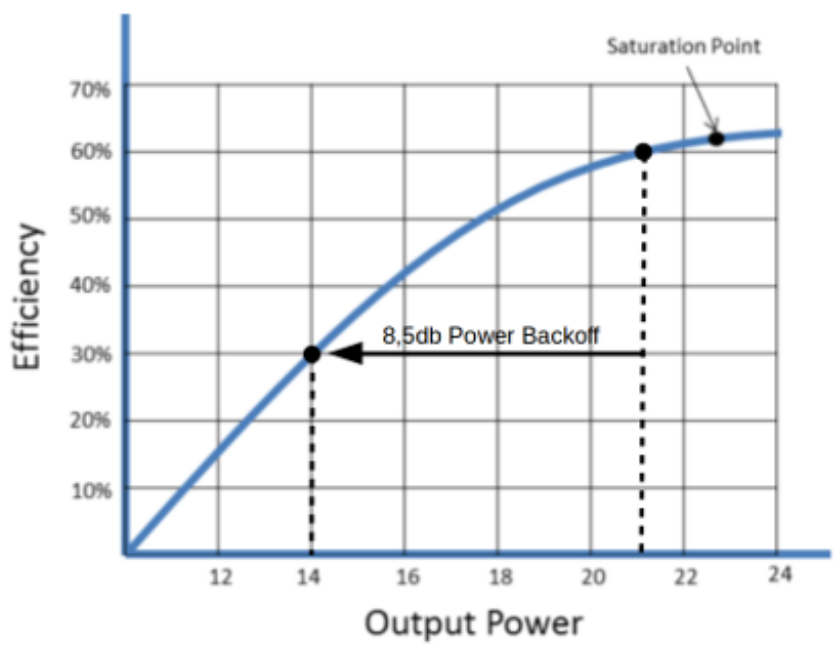

Fig. 2: Power amplifier efficiency with power back-off

the number of antennas $M, \gamma$, the power amplifier efficiency and the signal dynamic range.

We denote $r(\gamma)=\log _{2}(1+\operatorname{SQINR}(\gamma))$ and $r_{Z F}=$ $\log _{2}(1+\mathrm{SNR})$.

\section{ENERGY-EFFICIENCY EXPRESSION}

The energy consumption needed for (3) is assumed to be given by the sum of the energy consumed by the BS power amplifier $P_{T X}$ and by the BS circuits $P_{C P}$.

\section{A. Power amplifier energy consumption}

It is usually agreed that the power amplifier is the main source of power consumption, [14]. We will therefore carefully consider how to model it. For the one-bit quantized case, the power consumption of the power amplifier can be expressed as

$$
P_{T X}=\frac{B E\left[\|\mathbf{x}\|^{2}\right]}{\eta}=\frac{2 M B \rho}{\eta}
$$

where $\eta$ is the power amplifier efficiency at the BS [15]. The numerator of (6) equals the average transmitted power, which is exact since the signal's envelope is constant for QPSK signals. The power amplifier can hence operate near its saturation zone, so that the state-of-the art efficiency $\eta=0.6$ can be achieved.

The power amplifier efficiency $\eta$ is highly dependent on the signal dynamic range, usually measured by the peak-toaverage power ratio (PAPR), see [15]. For a signal with a high dynamic range, as $\mathbf{x}_{Z F}$, a power back-off $P_{B O}$ is used in order to keep the power amplifier output signal from saturating when $\left|x_{Z F, m}\right|$ is maximal, see Fig. 2 and [16]. Using the same power amplifier, this induces also an operating point much lower than the saturation zone so that the DC voltage from the power supply is also decreased, yielding an efficiency of only about $\eta_{Z F}=0.3$.

For the non-quantized case, the average power consumption of the power amplifier can be expressed as 
$P_{T X, Z F}=\frac{B E\left[\left\|\mathbf{x}_{Z F}\right\|^{2}\right]}{\eta_{Z F}} . E\left[\left\|\mathbf{x}_{Z F}\right\|^{2}\right]=\operatorname{trace}\left(E\left[\mathbf{x}_{Z F}^{H} \mathbf{x}_{Z F}\right]\right)=$ $2 \rho_{Z F} \operatorname{trace}\left(E\left[\left(\mathbf{H H}^{H}\right)^{-1}\right]\right)$ assuming independence between the symbols and the channel gains. For large enough values of $M$ and $K$, and finite $\gamma$, [7] shows that $\operatorname{trace}\left(E\left[\left(\mathbf{H H}^{H}\right)^{-1}\right]\right) \approx$ $\frac{K}{(M-K)} \zeta$ where $\zeta=\frac{1}{K} \sum_{k=1}^{K} \frac{1}{\sigma_{k}^{2}}$ is the average of the inverse variance of the channels of the different users. Here, $\zeta=\frac{1}{\sigma^{2}}$. Therefore, the average power consumption of the power amplifier can be approximated as

$$
P_{T X, Z F}=\frac{B E\left[\left\|\mathbf{x}_{Z F}\right\|^{2}\right]}{\eta_{Z F}} \approx \frac{2 B \rho_{Z F}}{\eta_{Z F}} \zeta \frac{1}{(\gamma-1)}
$$

Equation (7) is consistent with [12]. The differences are due to the power allocation and users' distribution.

Expressions (6) and (7) show the importance of the BS "load", $\frac{1}{\gamma}$. In order to have a fair BER comparison between the one-bit quantized and full dynamic range cases, [7] assumes that the signal power at the input of the power amplifier is equal, so that $\zeta=M(\gamma-1) \frac{\rho}{\rho_{Z F}}$. However, due to the power back-off, we propose to use instead the following

$$
P_{B O}\left[\text { in dB] }=10 \log (M \rho)-10 \log \left(\frac{\rho_{Z F} \zeta}{\gamma-1}\right)\right.
$$

where the power back-off is chosen to compensate for the $\mathrm{ZF}$ signal PAPR, as in Fig. 2.

\section{B. Circuit power consumption}

We collect here the terms of energy consumed in the different analog and digital components of the BS and user circuits. Unlike many studies on MIMO systems, the energy consumption is not limited to $P_{F I X}$, the power consumption due to site-cooling, control signaling, and the load-independent power of backhaul infrastructure, etc. The work in [12] shows that the circuit power consumption depends not only on the load $K / M$, but also on the number of antennas at the base station, $M$, and the resulting cost of the signal processing can no longer be ignored. Assuming the power cost due to channel estimation can be neglected, the total power consumed by the BS hardware can be expressed as,

$$
P_{C P}=P_{F I X}+P_{T C}+P_{C / D}+P_{B H}+P_{L P}
$$

where

- $P_{T C}$ is the energy consumption in the BS and mobile units, which scales with the number of transmitters $M$ and users $K$ as:

$$
\begin{aligned}
P_{T C} & =M P_{B S}+P_{S Y N}+K P_{U E} \\
& =M\left(P_{B S}+\gamma P_{U E}\right)+P_{S Y N}
\end{aligned}
$$

with

- $P_{B S}$ the power required for the components of the transmitter circuit at each antenna of the BS (converters, mixers and filters),

- $P_{S Y N}$ the power of the local oscillator,

- $P_{U E}$ the power required for the components of the circuit of each single antenna user (amplifier, mixer, oscillator and filter).
- two terms, $P_{C / D}$ and $P_{B H}$, that are proportional to the data rate. $P_{C / D}$ corresponds to the channel encoding at the BS and decoding at the user end:

$$
\begin{aligned}
P_{C / D} & =r_{B}\left(P_{C O D}+P_{D E C}\right) \\
& \approx B \frac{M}{\gamma} r(\gamma)\left(P_{C O D}+P_{D E C}\right)
\end{aligned}
$$

where $P_{C O D}$ and $P_{D E C}$ are the power of encoding and decoding in Watts/bit/s (W/bit/s), respectively.

$P_{B H}$ is the power needed by the backhaul with respect to the data load:

$$
P_{B H}=r_{B} P_{B T} \approx B \frac{M}{\gamma} r(\gamma) P_{B T}
$$

where $P_{B T}$ is the power of the backhaul traffic in W/ bit/ s.

- $P_{L P}$ is the power consumed to perform the linear precoder processing at the $\mathrm{BS}$. In our case, it is the $\mathrm{ZF}$ precoding for both cases. The processing due to onebit quantization can be neglected compared with the precoding calculation. Using a Cholesky decomposition to perform the channel pseudo-inversion, [12] shows

$$
\begin{aligned}
P_{L P} & =B\left(\frac{K^{3}}{3 L_{B S}}+3 \frac{M K^{2}+M K}{L_{B S}}\right) \\
& =\frac{B M^{2}}{L_{B S}}\left(\frac{M}{3 \gamma^{3}}+\frac{3 M}{\gamma^{2}}+\frac{3}{\gamma}\right)
\end{aligned}
$$

where $L_{B S}$ is the arithmetic complex-valued operations per Joule (in [flops/W]).

\section{Asymptotic energy-efficiency expression}

Collecting the expressions from the previous equations, for the case of one-bit quantized ZF precoding we obtain, the expression as displayed in (14), at the top of the next page. If we consider only the power amplifier consumption, the energyefficiency in (14) reduces to $\frac{\eta r(\gamma)}{2 \rho \gamma}$ which depends only on $\gamma$.

For the ZF precoder, the expression is displayed in (15). If we consider only the power amplifier consumption, the energyefficiency in (15) reduces to $M \frac{\eta_{Z F} r_{Z F}(\gamma-1)}{2 \rho_{Z F} \zeta \gamma}$. Introducing the power back-off in (8), it becomes $\frac{\eta_{Z F} r_{Z F}}{2 \rho \gamma} 10^{\frac{P_{B O}}{10}}$ again depending only on $\gamma$. These rough approximations indicate that the main parameter for the energy efficiency is the system inverse load $\gamma=M / K$, and that the loss of performance of the one-bit quantized massive MIMO system could be compensated by the higher power amplifier efficiency of the quantized system and the power back-off of the unquantized system.

\section{Simulations}

The one-bit quantized massive MIMO is designed to be used for low to moderate SNR values, typically between -5 and $35 \mathrm{~dB}$. To ensure the data rate is large enough for the system to be useful, the $\operatorname{SQINR}(\gamma)$ must also be large enough, so in the first simulation, we assume the $\mathrm{SNR}=10 \mathrm{~dB}$. We set the noise variance on the real and imaginary received symbols as $\frac{\sigma_{n}^{2}}{2}=\rho \frac{1}{\mathrm{SNR}}$. In addition, we consider the same parameters as in [12]. $P_{B O}$ is chosen as in Fig. $2,8.5 \mathrm{~dB}$ being a realistic value 


$$
E E \approx \frac{M \frac{r(\gamma)}{\gamma}}{\frac{P_{F I X}+P_{S Y N}+M\left(P_{B S}+\frac{P_{U E}}{\gamma}\right)}{B}+\frac{2 M \rho}{\eta}+M \frac{r(\gamma)\left(P_{C O D}+P_{D E C}+P_{B T}\right)}{\gamma}+\frac{M^{2}}{L_{B S}}\left(\frac{M}{3 \gamma^{3}}+\frac{3 M}{\gamma^{2}}+\frac{3}{\gamma}\right)}
$$

$$
E E_{Z F} \approx \frac{M \frac{r_{Z F}}{\gamma}}{\frac{P_{F I X}+P_{S Y N}+M\left(P_{B S}+\frac{P_{U E}}{\gamma}\right)}{B}+\frac{2 \rho_{Z F}}{\eta_{Z F}} \frac{\zeta}{(\gamma-1)}+M \frac{r_{Z F}\left(P_{C O D}+P_{D E C}+P_{B T}\right)}{\gamma}+\frac{M^{2}}{L_{B S}}\left(\frac{M}{3 \gamma^{3}}+\frac{3 M}{\gamma^{2}}+\frac{3}{\gamma}\right)}
$$

\begin{tabular}{|c|c||c|c|}
\hline$B$ & $20 \mathrm{MHz}$ & $\rho$ & $1 \mathrm{~W} / \mathrm{MHz}$ \\
$P_{B O}$ & $8.5 \mathrm{~dB}$ & $\zeta=1 / \sigma^{2}$ & 1 \\
$P_{F I X}$ & $18 \mathrm{~W}$ & $P_{C O D}+P_{D E C}$ & $0.9 \mathrm{~W} / \mathrm{Gbit} / \mathrm{s}$ \\
$P_{S Y N}$ & $2 \mathrm{~W}$ & $P_{B T}$ & $0.25 \mathrm{~W} / \mathrm{Gbit} / \mathrm{s}$ \\
$P_{B S}$ & $1 \mathrm{~W}$ & $L_{B S}$ & $12.8 \mathrm{Gflops} / \mathrm{W}$ \\
$P_{U E}$ & $0.1 \mathrm{~W}$ & & \\
\hline$\eta$ & 0.6 & $\eta_{Z F}$ & 0.3 \\
\hline
\end{tabular}

TABLE I: Simulation parameters

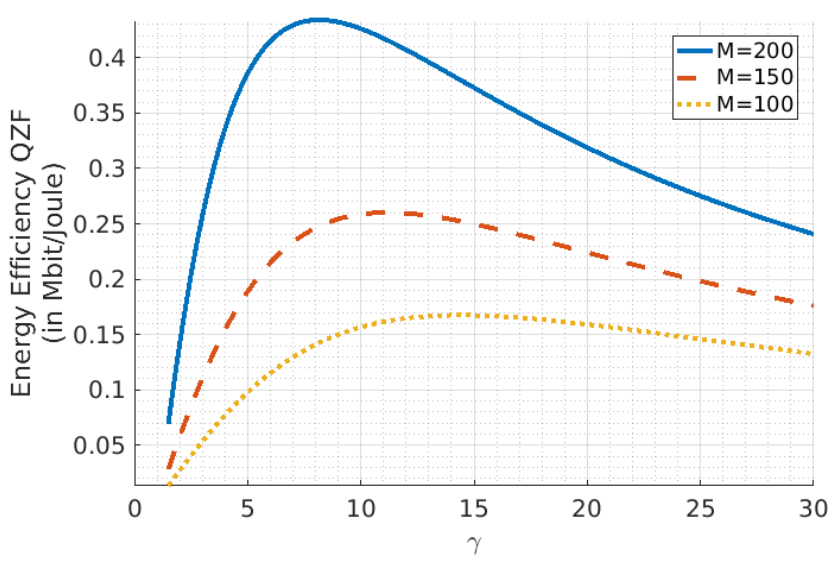

Fig. 3: Energy efficiency in [Mbit/Joule] of one-bit quantized $\mathrm{ZF}$ with respect to $\gamma$, for different values of $M, \mathrm{SNR}=10 \mathrm{~dB}$

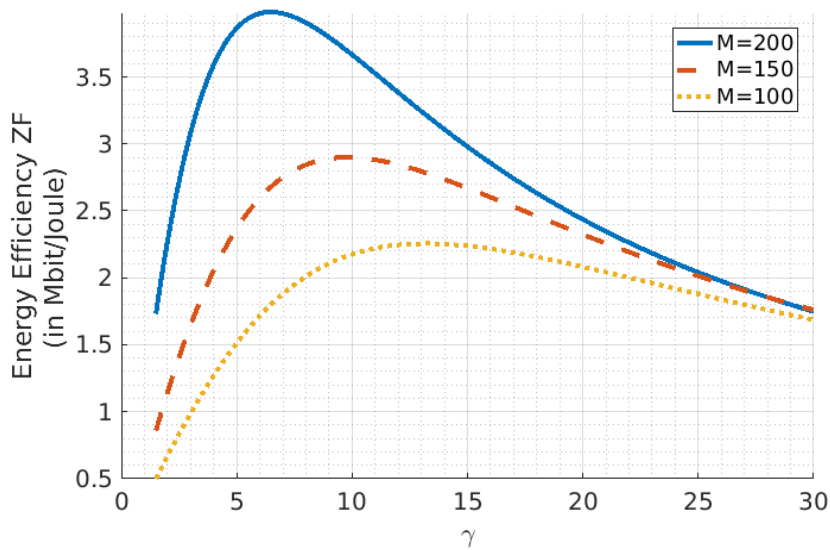

Fig. 4: Energy efficiency in [Mbit/Joule] of ZF with respect to $\gamma$, for different values of $M, \mathrm{SNR}=10 \mathrm{~dB}$

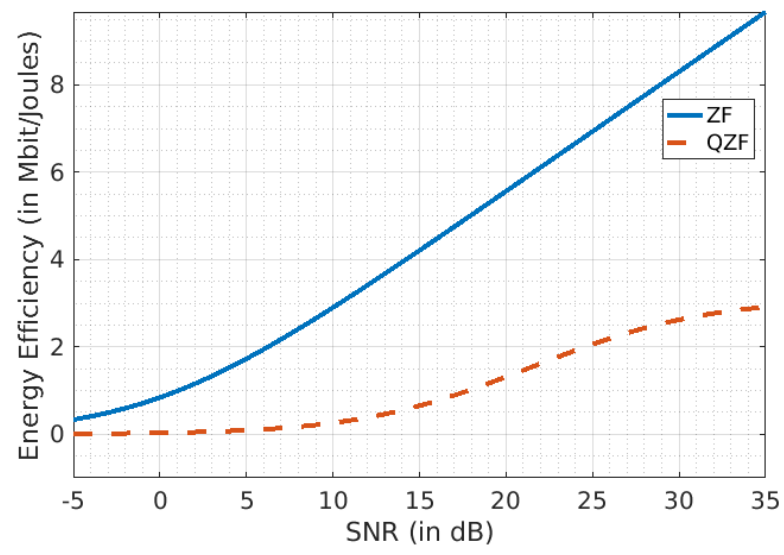

Fig. 5: Energy efficiency in [Mbit/Joule] with respect to SNR, for $M=150$ and $\gamma=10$

for LTE, [17]. From $P_{B O}$ and $\zeta$, we obtain $\rho_{Z F}$ satisfying (8). All values are collected in Table I.

Figs. 3 and 4 display the energy efficiency with respect to $\gamma$ for different values of $M$, at SNR $=10 \mathrm{~dB}$, for the one-bit quantized ZF precoder and for the full dynamic range signals respectively. They show that $\gamma$ is the crucial parameter. Both schemes have similar behavior with respect to $\gamma$. However, as with the BER performance in [7], the energy efficiency of the unquantized scheme is still better by a factor of 10 or higher, inspite of the power back-off taken.

On Fig. 5, we can see how this gap increases when SNR increases from -5 to $35 \mathrm{~dB}$ since the energy efficiency of the ZF increases dramatically with SNR. When we consider the ratio of these curves, the optimum for the one-bit quantized ZF precoder is achieved for a SNR around $30 \mathrm{~dB}$.

\section{CONClusion}

In this paper, we conducted a preliminary study of the energy efficiency of downlink multi-user one-bit quantized massive MIMO systems. This study focuses on the ZF precoder, but the approach can be extended to any precoder. We quantified the advantage of the one-bit quantization which allows the power amplifier to perform at its best power efficiency and with no need of power back-off to reduce the high signal dynamic range induced by the precoding. The analysis shows the inverse system load $\gamma=M / K$ is a crucial parameter, but the specific number of antennas $M$ can not be ignored. Further analysis should include the power used for 
the uplink transmission, the channel estimation and a more realistic evaluation of the signal PAPR as in [18].

\section{ACKNOWLEDGMENT}

This research was supported in part by the Orange Chair on IoT within the Fondation of University of Cergy-Pontoise, by the French National Research Agency (ANR) project ELIOT ANR-18-CE40-0030 and FAPESP 2018/12579-7 project and by the National Science Foundation under Grants ECCS1547155 and CCF-1703635.

\section{REFERENCES}

[1] E. Larsson, O. Edfors, F. Tufvesson, and T. Marzetta, "Massive MIMO for next generation wireless systems", IEEE Communication Magazine, vol. 52, no. 2, pp. 186-195, Feb 2014.

[2] H. Yang and T. Marzetta, "Performance of Conjugate and Zero-Forcing Beamforming in Large-Scale Antenna Systems", IEEE Journal on Selected Areas in Communications, vol. 31, no. 2, pp. 172-179, Feb 2013.

[3] D. Ha, K. Lee and J. Kang, "Energy efficiency analysis with circuit power consumption in massive MIMO systems", in 2013 IEEE 24th Annual International Symposium on Personal, Indoor, and Mobile Radio Communications (PIMRC), London, United Kingdom, pp. 938-942, 2013.

[4] J.A. Nossek, and M.T. Ivrlac, "Capacity and coding for quantized MIMO systems", in Proceedings of the 2006 international conference on Wireless communications and mobile computing, Vancouver, Canada, pp. 1387$1392,2006$.

[5] Y Li, C Tao, AL Swindlehurst, A Mezghani, L Liu, "Downlink achievable rate analysis in massive MIMO systems with one-bit DACs", IEEE Communications Letters, 21 (7), 1669-1672, 2017.

[6] S Jacobsson, G Durisi, M Coldrey, U Gustavsson, C Studer, "Throughput analysis of massive MIMO uplink with low-resolution ADCs", in IEEE Transactions on Wireless Communications, 16 (6), 4038-4051, 2017.

[7] Amodh Kant Saxena, Inbar Fijalkow, and A. Lee Swindlehurst, "Analysis of One-Bit Quantized Precoding for the Multiuser Massive MIMO Downlink", IEEE Transactions on Signal Processing, Vol. 65, N. 17, September, 2017

[8] Y Li, C Tao, G Seco-Granados, A Mezghani, AL Swindlehurst, L Liu, "Channel estimation and performance analysis of one-bit massive MIMO systems" IEEE Transactions on Signal Processing, 65 (15), 4075-4089, 2017.

[9] K. Roth, H. Pirzadeh, A. L. Swindlehurst and J. A. Nossek, "A Comparison of Hybrid Beamforming and Digital Beamforming With LowResolution ADCs for Multiple Users and Imperfect CSI," in IEEE Journal of Selected Topics in Signal Processing, vol. 12, no. 3, pp. 484-498, June 2018.

[10] H. Pirzadeh and A. L. Swindlehurst, "Spectral Efficiency under Energy Constraint for Mixed-ADC MRC Massive MIMO," in IEEE Signal Processing Letters, vol. 24, no. 12, pp. 1847-1851, Dec. 2017.

[11] H.Q. Ngo, E.G. Larsson, T.L. Marzetta, "Energy and spectral efficiency of very large multiuser MIMO systems", IEEE Transactions on Communications, 61 (4), April 2013.

[12] Emil Bjornson, Luca Sanguinetti, Jakob Hoydis, and Merouane Debbah, "Optimal Design of Energy-Efficient Multi-User MIMO Systems: Is Massive MIMO the Answer?", IEEE Transactions on Wireless Communications, 14 (6), pp.3059-3075, 2015.

[13] Emre Telatar, "Capacity of multi-antenna Gaussian channels", in European transactions on telecommunications, 10 (6), 585-595, 1999.

[14] H Holtkamp, G Auer, V Giannini, H Haas, "A parameterized base station power model", in IEEE Communications Letters, 17 (11), 2033-2035, 2013.

[15] J.-L. Gautier, Myriam Ariaudo and Cédric Duperrier, "Power amplification" in Design of Microwave Active Devices, edited by J.-L. Gautier, Wiley, 2014.

[16] Ralf R. Müller, Mohammad A. Sedaghat and Georg Fische, "Load Modulated Massive MIMO" in proc. IEEE Global Conference on Signal and Information Processing (GlobalSIP), 2014.

[17] A. Ghosh, R. Ratasuk, B. Mondal, N. Mangalvedhe, T. Thomas, "LTEAdvances : Next-Generation wireless broadband technology", IEEE Wireless Communications, 17(3), June 2010.

[18] D. Persson, T. Eriksson, E. G. Larsson, "Amplifier-aware multiple-input multiple-output power allocation." in IEEE Communications Letters, vol. 17, no 6, p. 1112-1115, 2013. 